\title{
Оптическая прозрачность графеновых слоев, выращенных на поверхности металлов
}

\author{
(C) Е.В. Рутьков ${ }^{1,2}$, Н.П. Лавровская ${ }^{2}$, Е.С. Шешеня ${ }^{1, \text { Ф Н.Р. Галль }}{ }^{1,3}$ \\ ${ }^{1}$ Физико-технический институт им. А.Ф. Иоффе Российской академии наук, \\ 194021 Санкт-Петербург, Россия \\ ${ }^{2}$ Государственный университет аэрокосмического приборостроения, \\ 190000 Санкт-Петербург, Россия \\ ${ }^{3}$ Институт аналитического приборостроения Российской академии наук, \\ 198095 Санкт-Петербург, Россия \\ ` E-mail: sheshenayket@gmail.com
}

(Получена 5 октября 2016 г. Принята к печати 14 октября 2016 г.)

Показано, что графеновые пленки, образованные на поверхности родия (111), при толщинах от одного слоя до $\sim(12-15)$ слоев не поглощают излучаемое поверхностью электромагнитное излучение в видимой области и не влияют ни на яркостную, ни на истинную температуру образца, в противоречии с фундаментальными результатами для свободного графена. При больших толщинах пленок поглощение имеет место. Данный эффект наблюдается и на поверхности других металлов, $\operatorname{Pt}(111), \operatorname{Re}(10 \overline{1} 0)$ и $\mathrm{Ni}(111)$, т.е. носит универсальный характер. На наш взгляд, эффект связан с изменением электронных свойств тонких слоев графена за счет перетекания электронов между графеном и металлической подложкой.

DOI: $10.21883 /$ FTP.2017.04.44345.8422

\section{1. Введение}

Удивительные оптические свойства однослойного графена явились предметом детального изучения в течение последнего десятилетия $[1,2]$. Несмотря на моноатомную толщину, свободный однослойный графен поглощает довольно большую долю света $\pi \alpha \approx 2.3 \%$, где $\alpha=e^{2} /(c h)$ - постоянная тонкой структуры [3-5]. Поэтому имеется возможность увидеть даже однослойный графен без помощи микроскопа.

Однако, как показано в нашей работе [6], графен на поверхности родия ведет себя по-другому, в частности он не поглощает свет: его образование при малой толщине никак не влияет на температуру нагретого металла. В частности, в согласии с $[3,4]$, можно предполагать, что при переходе от однослойного к многослойному графену, а затем к графиту вклады от каждого из слоев в поглощение света должны суммироваться, так как слои графена в графите связаны слабо, практически без электронного обмена. В то же время в описанной выше работе графен сохранял свою прозрачность вплоть до нескольких слоев, а затем начиналось поглощение. Целью настоящей работы является более детальное изучение этого эффекта на различных металлах и попытка обсуждения его феноменологической модели. Родий был выбран как металл, позволяющий быстро и воспроизводимо выращивать на поверхности графеновые и графитовые слои в широком диапазоне толщин - от субмонослойных до несколько десятков слоев. В соответствии с принятой на сегодня традицией мы используем термин „графен“ не только для монослойной пленки, но и для нескольких слоев в переходной области от однослойного „классического“ графена к графиту.

\section{2. Техника и методы эксперимента}

Опыты проводились в условиях сверхвысокого вакуума (остаточное давление $p \sim 10^{-10}$ Торр) в ожеспектрометре с энергетическим разрешением $\Delta E / E \sim 0.1 \%$ [7]. Образцами служили металлические ленты из Rh, Pt, Ir, Re и Ni размером $40 \times 1 \times 0.02 \mathrm{Mм}$, которые текстурировались прямым прогревом переменным током и очищались от примесей выдержкой нагретых лент в атмосфере кислорода и в сверхвысоком вакууме. После очистки ориентация поверхности лент Rh, Pt и Ir в направлении (111) составляла 99.8\%, для $\mathrm{Ni}$-ленты - 99.55\%. Средний размер зерен равнялся 40-50 мкм. В случае Re на поверхность выходила грань $(10 \overline{1} 0)$. Поверхность всех образцов была однородной по работе выхода с точностью 0.05 эВ. В камере прибора был собран узел для измерения тока термоэлектронной эмиссии и поверхностной ионизации зондирующих атомов с нагретых образцов. Оже-спектры могли сниматься прямо с нагретых вплоть до $1800 \mathrm{~K}$ металлических лент.

Образцы науглероживались по стандартной процедуре - выдержкой нагретых лент в изотермическом режиме в атмосфере бензола до достижения момента, когда углерод насыщал объем образца до предельной растворимости, а на поверхности образовывался монослой графена. Детально процедура науглероживания родия обсуждается в работе [8], а для других металлов $(\mathrm{Re}, \mathrm{Pt}, \mathrm{Ni}, \mathrm{Ir}$ и т.д.) - в работе [7]. Малая толщина ленты обеспечивала быстроту диффузионных процессов и достижение реального термодинамического равновесия в области температур выше $1100-1200 \mathrm{~K}$ за время порядка десятка секунд. После этого пары бензола откачивались, а на поверхности металла оставался строго моноатомный слой графена. 
B процессе науглероживания атомы C проникают в объем ленты и, вообще говоря, могут изменить ее электрическое сопротивление, а тем самым при постоянном токе накала и выделяемую в нем мощность. Однако за счет низкой предельной растворимости атомов С в $\mathrm{Rh}$ этот эффект лежит за пределами точности измерений. Так, в интервале температур науглероживания $T_{\mathrm{C}}=1200-1800 \mathrm{~K}$ прирост мощности за счет изменения сопротивления составил $<1 \%$, а яркостная температура ленты оставалась постоянной с точностью, которую обеспечивает оптический пирометр $(\sim 5 \mathrm{~K})$. Теплопроводность держателей ленты выбиралась по возможности малой. При этом в пирометрической области ее средняя часть длиной $\sim 35$ мм однородна по температуре с той же точностью.

\section{3. Образование пленок графена и графита на поверхности родия}

Если температуру родия, науглероженного при $T_{\mathrm{C}}$, понизить, то твердый раствор углерода в металле становится пересыщенным, и избыточный углерод выходит на поверхность, образуя графеновые и графитовые слои. Как при монослойной, так и при полислойной толщине пленка имеет графеновую (графитовую) структуру, что подтверждается измерениями с помощью сканирующей туннельной микроскопии (СТМ) $[9,10]$, рамановского рассеяния света [11], электронной ожеспектроскопией [12,13].

На рис. 1 (кривые 1 и 2) представлена кинетика изменения оже-сигналов углерода (энергия $E=272$ эВ) и подложки (энергия $E=302$ эВ) при выделении углерода из объема на поверхность родия, науглероженного при $T_{\mathrm{C}}=1360 \mathrm{~K}$ и выдержанного при температуpe $T=1260 \mathrm{~K}$, а кривая 3 - это изменение работы выхода $(e \varphi)$ системы в процессе роста графена (работу выхода измеряли по полному току термоэлектронной эмиссии) [13]. Оже-сигнал углерода растет и выходит на плато при толщине пленки, в несколько раз превышающей длину свободного пробега оже-электронов данной энергии $(\lambda \sim 5 \AA)[12]$. При этом одновременно перестает регистрироваться сигнал подложки из-за экранирования углеродом. Форма оже-спектра углерода на всем протяжении роста пленки типична для графена (рис. 1) [10].

Известно, что один слой графена уменьшает ожесигнал подложки в 1.6 раза при энергии электронов $150-300$ эВ $[10,13]$. В этом случае можно определить время образования второго слоя графена, третьего и т.д. по уменьшению оже-сигнала родия (энергия $E=302$ эВ). Графеновая пленка растет почти послойно, как и на ранее изученных Re, Pt и Ni $[10,13]$. Более того, оказалось, что время образования каждого последующего слоя графена практически одинаково; это означает, что диффузионные процессы в объеме достаточно быстры, а рост лимитируется выходом атомов

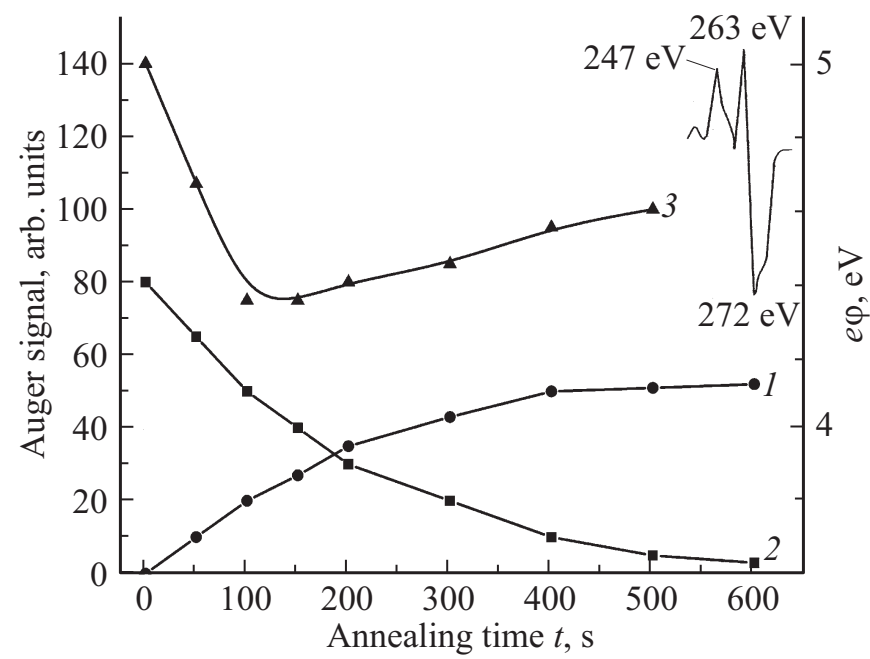

Рис. 1. Зависимость оже-сигнала углерода (1) и родия (2) от времени $t$ прогрева родиевой ленты при $1260 \mathrm{~K}$. Лента науглерожена при $T_{\mathrm{C}}=1360 \mathrm{~K} .3$ - изменение работы выхода $е \varphi$. На вставке - оже-спектр углерода для $t>500 \mathrm{c}$.

C на поверхность и нуклеацией нового слоя графена. Зондирование пленки графена на родии молекулами $\mathrm{CsCl}$ по степени диссоциации молекул (регистрируются ионы $\mathrm{Cs}^{+}$) указывает, что пленка графена сплошная, а дефектные места в слое составляют не более $10^{-3}$ от всей поверхности пленки [8].

\section{4. Расчет толщины графеновой и графитовой пленки}

Толщина (число слоев $k$ ) графеновой и графитовой пленки, образующейся на поверхности при понижении температуры, определяется разностью предельных растворимостей $\mathrm{C}$ в $\mathrm{Rh}$ при температуре науглероживания, $n_{\mathrm{C}}\left(T_{\mathrm{C}}\right)$, и при температуре роста пленки, $n_{\mathrm{C}}(T)$,

$$
k=\left[n_{\mathrm{C}}\left(T_{\mathrm{C}}\right)-n_{\mathrm{C}}(T)\right] n_{\mathrm{Rh}} / 2 N_{\mathrm{CML}},
$$

где $n_{\mathrm{Rh}}-$ количество атомов родия в образце объемом $V=S h=1 \mathrm{~cm}^{2} \cdot 2 \cdot 10^{-3} \mathrm{~cm}=2 \cdot 10^{-3} \mathrm{~cm}^{3}$; $N_{\mathrm{CML}}=3.86 \cdot 10^{15} \mathrm{~cm}^{2}-$ концентрация атомов С в монослое графена; коэффициент $1 / 2$ связан с выделением углерода одновременно на обеих поверхностях ленточного образца. Величина $n_{\mathrm{C}}(T)$ была тщательно измерена нами в работе [13]. Выполненные расчеты хорошо согласуются с данными оже-спектроскопии при росте пленки малой толщины $(k<6)$, большие толщины пленок оже-спектроскопия не чувствует.

Метод вычисления толщины графеновой пленки из разницы предельных растворимостей надежен и работает на многих подложках. Так, аналогичные опыты по выделению углерода были проведены на поверхности $\mathrm{Ni}(111)$ из пересыщенного твердого раствора. 
Используя формулу (1) для случая никелевой ленты $\left(T_{\mathrm{C}}=1430 \mathrm{~K}\right.$ и $\left.T=1100 \mathrm{~K}\right)$, получили $k \approx 1000$, т.е. ожидаемая толщина графитовой пленки с каждой стороны ленты $h \approx 0.3$ мкм. После образования такой пленки никель растворялся в 65\%-й серной кислоте, отделившаяся графитовая пленка переносилась на кремниевую подложку, и методом атомно-силовой микроскопии определяли среднюю толщину такой пленки. Оказалось, $h=0.40$ мкм, что согласуется с расчетной ожидаемой толщиной, вычисленной по формуле (1). К сожалению, непосредственно на родии такие опыты выполнить невозможно, так как этот металл не растворяется в обычных кислотах, а растворение в царской водке заведомо повреждает графен.

\section{5. Измерение оптические свойства графеновых пленок на родии}

На рис. 2 представлено изменение яркостной (кривая 1) и истинной (кривая 2) температур родиевого образца при росте на его поверхности графена и графита. Из-за лавинообразного процесса выделения углерода при понижении температуры, описанного нами в работах $[8,10]$, эти кривые не могут быть сняты в прямом опыте. Для снятия этих кривых опыт ставился следующим образом. Образец последовательно науглероживался при все более высоких температурах в интервале $1260-1800 \mathrm{~K}$. После каждого цикла науглероживания пары бензола откачивались, а протекающий через образец ток скачком понижался до величины, соответствующей образованию пленки необходимой толщины, рассчитываемой по формуле (1). Как и следовало ожидать, при повышении $T_{\mathrm{C}}$ максимальная толщина выросшей пленки оказывалась все большей. После достижения стационарного состояния измеренные температуры наносились на график. Отметим, что электрическая мощность, выделяющаяся в образце, не менялась в процессе роста графена, и каждый раз совпадала с мощностью, рассеивающейся в ненауглероженной ленте при этом же токе накала.

Яркостная температура измерялась цветовым пирометром Piro на длине волны 580 нм. Как видно из рис. 2, при росте графитовой пленки толщиной до 15 слоев она остается неизменной. При времени, отвечающем $12-15$ слоям, она начинает уменьшаться, сначала незначительно, потом все сильнее. При $\sim 100$ слоях такое уменьшение соответствует $\sim 300 \mathrm{~K}$. Далее кривая стабилизируется, и при дальнейшем росте толщины пленки температура остается неизменной.

Изменение истинной температуры нельзя напрямую рассчитать из пирометрических данных, так как в процессе роста графена сильно меняется коэффициент серости. Неэффективной оказывается и многолучевая пирометрия, так как для устойчивости расчетов требуются точные значения коэффициентов серости на

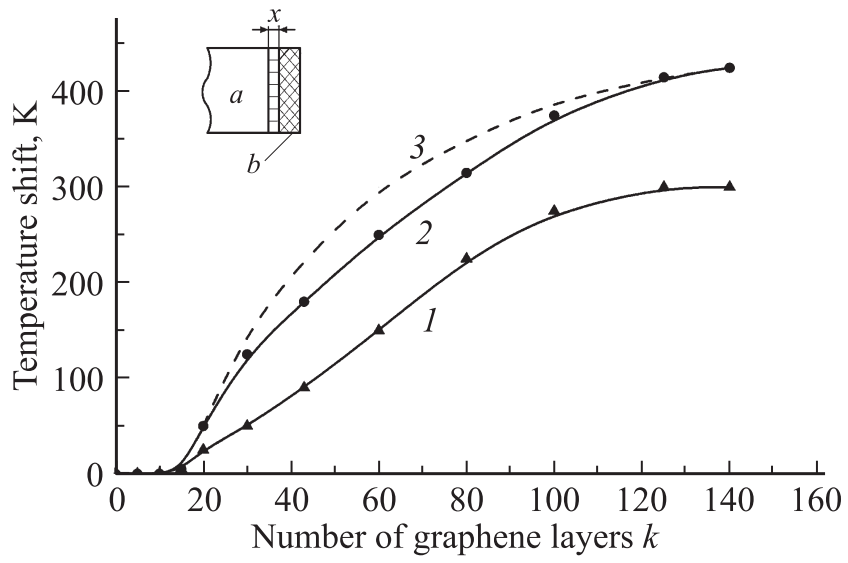

Рис. 2. Изменение яркостной $(1)$ и истинной (2) температур родиевых образцов в зависимости от числа $k$ слоев графена на его поверхности. 3 - расчетная кривая. На вставке - модельное представление: $a$ - родиевый образец, $b-$ графитовая пленка, с переходной областью толщиной $x$.

разных длинах волн, в отношении которых в литературе имеется очень существенный разброс.

В наших экспериментах температура определялась методом, предложенным и верифицированным нами в [14], на основе одновременного измерения тока термоэлектронной эмиссии и тока поверхностной ионизации трудно ионизуемого элемента (Na). Как показано в [14], метод позволяет измерять абсолютную температуру с высокой точностью, при этом не требуется знание такого параметра, как работа выхода пленки. Абсолютная температура находится из уравнения

$$
I^{-} I^{+}=B T^{2} \exp \left[-V / k_{\mathrm{B}} T\right]
$$

где $I^{-}$и $I^{+}$- токи термоэлектронный и поверхностной ионизации $\mathrm{Na}^{+}, V-$ потенциал ионизации натрия, $k_{\mathrm{B}}$ - постоянная Больцмана; коэффициент $B=A_{\mathrm{R}} A(1-R) S^{2} e v$, где $e-$ заряд электрона, $A_{\mathrm{R}}=150 \mathrm{~A} / \mathrm{M}^{2} \cdot \mathrm{K}^{2}$ - постоянная Ричардсона для графита, $v$ - поступающий на поверхность поток атомов $\mathrm{Na}$, $A=Q_{0} / Q_{+}$- отношения статсумм для атома и иона натрия, $S$ - площадь сбора токов, $R-$ коэффициент обратного отражения электронов (обычно принимают $R=0)$.

Другим методом определения температуры было измерение уменьшения тока термоэлектронной эмиссии при росте толщины слоя. Этот ток уменьшается на 5 порядков и описывается формулой Ричардсона

$$
I^{-}=S A T^{2} \exp [-e \varphi / k T]
$$

где $е \varphi$ - работа выхода для пленки заданной толщины, прочие обозначения см. выше. Обеспечивая постоянство площади сбора $S$, можно измерить истинную температуру с высокой точностью, но необходимо также знать величину работы выхода. 
Для монослойного графена работу выхода измеряли методом Ричардсона, она составила $(4.35 \pm 0.05)$ эВ. Для многослойной пленки с $k>80-100$, когда яркостная температура $T_{B}$ перестает зависеть от толщины, это делалось тоже методом Ричардсона, поскольку при

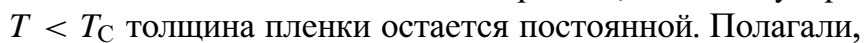
что ее оптические характеристики соответствуют таковым для объемного графита, т.е. коэффициент серости $\varepsilon=0.8$, и для расчета истинной температуры использовали общепринятую формулу [15]

$$
1 / T_{B}-1 / T=4.62 \cdot 10^{-5} \ln (1 / \varepsilon) .
$$

Это позволяет построить график Ричардсона для многослойной пленки и с большой точностью рассчитать ее работу выхода: $е \varphi=(4.75 \pm 0.05) \ni \mathrm{B}$.

При промежуточных толщинах графеновой пленки работу выхода определяли методом полного тока; ее значения приведены в виде кривой 3 рис. 1. Как видно, работа выхода резко падает от величины 5.0 эВ, соответствующей чистому родию, до 4.35 эВ, соответствующей одному монослою графена, а затем медленно растет. Измерения при более высоких температурах науглероживания, а значит, и бо́льших толщинах пленок показывают, что $е \varphi$ достигает значения $4.75 \mathrm{\jmath B}$, характерного для многослойной пленки, при толщинах $>15$ слоев графена.

Оба метода измерения абсолютной температуры дали значения, различающиеся в пределах $<10 \mathrm{~K}$, что показывает высокую точность измерения работы выхода. На кривой 2 рис. 2 показано изменение истинной температуры образца при росте толщины графитовой пленки. Отметим, что при максимальной толщине пленки истинная температура уменьшилась на $\Delta T \approx 430 \mathrm{~K}$, как это ранее наблюдалось в [16].

Рассмотрим пример получения данных, пусть температура науглероживания $T_{\mathrm{C}}=1750 \mathrm{~K}, n_{\mathrm{C}}=0.4 \mathrm{aT} \%$ и на поверхности имеется монослой графена. Понизим ток нагрева скачком с 3.5 до $3.0 \mathrm{~A}$, чему соответствовала бы температура $T_{1}=1450 \mathrm{~K}$ в случае ненауглероженной ленты. Избыточный углерод из объема сегрегирует на поверхность, образуя многослойную графеновую пленку. Это приводит к росту коэффициента серости, а значит, и радиационного сброса тепла, что в свою очередь приводит к уменьшению яркостной и истинной температур, а также тока термоэлектронной эмиссии, даже при постоянстве работы выхода. После завершения процесса вычисляем новую истинную температуру по формуле (2), $T_{2}=1350 \mathrm{~K}$, и определяем соответствующую ей предельную растворимость $n_{\mathrm{C}}=0.06$ ат\%. Используя формулу (1), легко оценить число слоев в графитовой пленке: $k=30$, при этом $\Delta T=T_{1}-T_{2}=1450-1350 \mathrm{~K}=100 \mathrm{~K}$. Чтобы получить более толстую пленку, родий науглероживали при более высокой температуре.

Таким образом, из рис. 2 следует, что при толщинах графеновой пленки $k<(12-15)$ слоев ни истинная, ни яркостная температура системы не меняются в согласии с результатами, полученными нами ранее в [6], т.е. система как бы „не замечает“ наличия на поверхности слоев графена.

\section{6. Обсуждение результатов}

В проводимых экспериментах нами были приняты меры, минимизирующие уход тепла на держатели, и в рассматриваемой высокотемпературной области можно смело им пренебречь, во всяком случае для центральной части ленты, что гарантируется ее температурной однородностью. Поэтому выделяющаяся в образце электрическая мощность полностью излучается с поверхности, откуда следует

$$
I U=\varepsilon \sigma T^{4},
$$

где $I$ и $U-$ ток, протекающий через ленту, и падающее на ней напряжение, $\varepsilon-$ коэффициент серости в области максимума излучения $(\sim 580$ нм$), \sigma-$ постоянная Стефана-Больцмана, $T$ - истинная температура. В эксперименте левая часть равенства (выделяющаяся мощность) поддерживалась постоянной. По мере роста графена и графита коэффициент серости меняется от величины $\varepsilon=0.24$, соответствующей чистому металлу, до $\varepsilon=0.8$, соответствующей графиту. Одновременно менялась и яркостная температура $T_{B}$, связанная с истинной температурой $T$ соотношением (4).

Можно сделать предположение, что при малых толщинах растущий графен имеет тот же коэффициент серости, что и родий: тогда его рост действительно никак не скажется на температуре системы. Однако это справедливо лишь при очень точном совпадении коэффициента серости растущей пленки и подложки (в данном случае родия). Легко оценить, что даже ничтожные различия в их величинах, скажем в $1 \%$, уже приведут к значительному, абсолютно измеримому расхождению и в яркостной, и в истинной температурах для сравниваемых систем. Значит, надо верить, что растущие слои графена с очень высокой точностью соответствуют родию по эмиссионным свойствам, что теоретически возможно, но крайне маловероятно. Более того, эффект наблюдается и на других металлах (см. далее разд. 7), сильно отличающихся от родия по коэффициенту серости - уж совсем невероятно, чтобы на каждой подложке растущий графен идеально подстраивался под ее эмиссионные свойства.

Единственное физичное объяснение - это предположение, что по какой-то причине графен на поверхности не поглощает оптическое излучение и многослойная пленка остается оптически прозрачной вплоть до достижения некоторой толщины, после чего она начинает поглощать и переизлучать свет. Рассмотренное явление имеет достаточно общий характер и проявляется на всех исследованных металлах. 
На наш взгляд, оно связано с изменением электронных свойств тонких слоев графена за счет взаимодействия с металлической подложкой, на что указывают опыты по измерению работы выхода графеновых пленок. Так, например, работа выхода графена на родии перестает меняться и достигает значения, характерного для многослойной пленки как раз при тех толщинах, начиная с которых графеновая пленка перестает быть прозрачной. Аналогичная ситуация имеет место и на Re, где работа выхода однослойного графена $(k=1) e \varphi=4.25$ эВ и плавно меняется до значения $e \varphi=4.75$ эВ при $k \geq 10$; при бо́льших толщинах работа выхода перестает изменяться, но начинает уменьшаться температура образца. Кроме того отметим, что оптические свойства тонких графеновых пленок на разных металлах одинаковы, а работа выхода слоя графена разная $[10,13]$, но она всегда ниже, чем работа выхода многослойной пленки или базисной плоскости (0001) монокристалла графита.

Развитые представления подтверждаются данными, как теоретическими, так и экспериментальными, полученными в отношении свободного графена. Так, при образовании графена на металлах, имеющих большую концентрацию свободных электронов, возможно перетекание их в валентную зону графена, что приведет к сдвигу положения уровня Ферми и изменению оптического поглощения графена за счет действия принципа Паули [17]. Так как плотность электронных состояний в графене вблизи уровня Ферми относительно невелика, то даже электростатического допирования может оказаться достаточно, чтобы сдвинуть уровень Ферми на сотни мэВ [18]. Как утверждают лауреаты Нобелевской премии А. Гейм и К. Новоселов, сильное допирование для нескольких слоев графена, возможно, позволит регулировать прохождение света через такие пленки, что перспективно для создания новых устройств фотоники [2].

\section{7. Графен на других подложках}

Полученные результаты по неизменности температуры родиевого образца с пленками графена при малых толщинах $(k<15)$ заставили проверить это наблюдение для других металлических подложек - $\operatorname{Ir}(111)$, $\operatorname{Pt}(111), \operatorname{Re}(1 \overline{1} 0)$ и $\mathrm{Ni}(111)$. Графен на этих подложках образовывали, как и на $\mathrm{Rh}$, методом химического осаждения паров бензола. Взаимодействие указанных выше металлов с углеродом детально изучено нами в работах $[10-13,19]$. Максимальное число слоев графена на иридии составляло $k=1$, а на платине из-за малой предельной растворимости $k=10$ [10]. На рении возможно образование многослойной пленки, но диффузионные процессы с участием углерода идут в этой системе существенно медленнее, чем в случае родия: если на родии пленка графена для $k=50$ вырастает за секунды (десятки секунд), то на рении для этого требовалось $>3$ ч. На никеле, имеющем большую предельную растворимость, можно получить графеновые пленки в несколько сот атомных слоев [10].

Для всех изученных подложек рост графена вплоть до толщин $k \leq(10-15)$ слоев не приводил к изменению ни яркостной, ни истинной температуры. При больших толщинах максимальное изменение истинной температуры $\Delta T_{\max }$ зависело от соотношения $\varepsilon_{1}$ и $\varepsilon_{2}$. Так, в случае графена на $\mathrm{Ni}(111)$, имеющем $\varepsilon_{1}=0.36$ [17], величина $\Delta T_{\max }$ составила $\sim 200 \mathrm{~K}$.

\section{8. Простейшая расчетная модель излучения для пленки графита на родии}

Рассмотрим следующую интуитивно очевидную модель процесса излучения с поверхности родия, на которой имеются графеновые слои, и оценим ее валидность. С учетом слабой межслоевой связи можно предположить, что каждый из слоев полислойной пленки поглощает свет независимо, поэтому коэффициент оптического поглощения такой системы может быть рассчитан относительно просто.

Если имеется чистая поверхность родия, то интенсивность излучения будет равна $I_{\mathrm{Rh}}=\varepsilon_{1} I_{0}$, где $I_{0}-$ интенсивность излучения абсолютно черного тела, а $\varepsilon_{1}$ - коэффициент серости чистого родия. Эта излученная энергия при наличии слоев графена будет частично ими поглощаться, а частично проходить сквозь слои. Обозначим коэффициент поглощения одного слоя графена $A$, и, как говорилось выше, для свободного графена $A=0.023$, тогда прошедшая через единичный слой графена доля излучения равна $B=1-A$, т.е. 0.977 для свободного графена. При наличии на поверхности $k$ слоев графена послойное пропускаемое излучение будет равно $I_{\mathrm{tr}}=I_{\mathrm{Rh}} B^{k}$, а поглощенное излучение равно $I_{\mathrm{abs}}=I_{\mathrm{Rh}}-I_{\mathrm{tr}}$, при условии, что все слои графена поглощают одинаково.

Поглощенное излучение переизлучается с поверхности графена с коэффициентом $\varepsilon_{2}>\varepsilon_{1}$, где $\varepsilon_{2}-$ коэффициент серости графита, т. е. эта энергия переизлучается в $\varepsilon_{2} / \varepsilon_{1}$ раза лучше, чем с поверхности родия. Тогда излученная энергия для системы родий-графен будет равна

$$
\begin{aligned}
I_{\mathrm{rad}} & =I_{\mathrm{tr}}-\left(\varepsilon_{2} / \varepsilon_{1}\right) I_{\mathrm{abs}}=I_{\mathrm{Rh}}\left[B^{k}\left(1-\varepsilon_{2} / \varepsilon_{1}\right)+\varepsilon_{2} / \varepsilon_{1}\right] \\
& =\varepsilon_{1} I_{0}\left[B^{k}\left(1-\varepsilon_{2} / \varepsilon_{1}\right)+\varepsilon_{2} / \varepsilon_{1}\right]=\varepsilon_{\mathrm{eff}} I_{0} .
\end{aligned}
$$

Здесь вводится эффективный коэффициент серости $\varepsilon_{\text {eff }}$, который меняется между двумя предельными значениями $\varepsilon_{1}$ и $\varepsilon_{2}$.

Оценим, как меняется истинная температура родия при росте графеновых слоев на нем в рамках описанной выше модели. Из закона Стефана-Больцмана 
следует, что

$$
T=T_{\mathrm{Rh}}\left(\varepsilon_{1} / \varepsilon_{\mathrm{eff}}\right)^{0.25},
$$

где $T_{\mathrm{Rh}}-$ температура поверхности чистого родия до начала выделения графена. При расчете принимали $\varepsilon_{1}=0.24$, а $\varepsilon_{2}=0.8$ [19] с учетом того, что практически вся подводимая к образцу энергия уходит с него в виде излучения.

Рассмотрим, что дает нам описанная выше модель в применении к экспериментальным условиям, представленным на рис. 2. Для этого выберем в качестве начальной температуры $T=1600 \mathrm{~K}$. Так, всего 10 слоев графена должны были бы существенно уменьшить температуру родиевого образца, на $\Delta T \approx 150 \mathrm{~K}$, что полностью противоречит эксперименту. Однако при $k \geq 100$ рассчитанная температура изменяется на $\Delta T \approx 400 \mathrm{~K}$ и практически перестает уменьшаться с дальнейшим ростом числа слоев графена, что, напротив, очень хорошо коррелирует с экспериментом (рис. 2). Отметим, что при $k \geq 100$ излучательная способность системы родийграфен определяется только графеном, а точнее тонкой пленкой графита.

Таким образом, данная модель никак не описывает ситуацию, имеющую место при 1-10 слоях графена на поверхности. Этого и следовало ожидать, ведь в нее не заложены экспериментально полученные данные о прозрачности этих первых слоев.

Для согласования модели с экспериментом изменим ее, предположив, что имеется несколько прозрачных слоев графена толщиной $x$ в области, непосредственно примыкающей к поверхности металла, как это представлено на вставке к рис. 2. Толщина этого прозрачного слоя с $k=x$ нам заранее не известна, и мы можем просто взять ее из экспериментальных данных, скажем из кривой 1, полагая, что дальнейший рост пленки не влияет на прозрачность этих слоев, примыкающих к металлу. Таким образом, первые $x$ слоев графена мы полагаем прозрачными, а все последующие - поглощающими так, как это следует из теории Гейма [1]. Рассчитанная так кривая представлена на рис. 2 (кривая 3). Как видно, качественно она вполне корректно аппроксимирует кривую 2, характеризующую сдвиг истинной температуры системы при росте графена, хотя в области промежуточных значений толщин пленки имеются расхождения $\sim(15-20) \%$, по-видимому, связанные с тем, что графеновые слои не переходят мгновенно от состояния „прозрачности“ к состоянию „,пглощения света по Гейму“.

\section{9. Заключение}

Таким образом, показано, что тонкие графеновые слои в интервале толщин 1-10 слоев на поверхности переходных металлов ведут себя так, как будто они полностью прозрачны для оптического излучения, причем этот эффект наблюдается на различных подложках. При больших толщинах пленка начинает поглощать излучение. Модель, основанная на предположении, что первые 10 слоев графена оптически прозрачны, а все последующие поглощают свет в соответствии с теоретическим представлениями, качественно неплохо описывает экспериментальные зависимости истинной температуры от толщины пленки на поверхности родия. Мы связываем указанный эффект с перетеканием электронов между металлом и графеновой пленкой, что косвенно подтверждается измерениями работы выхода.

\section{Список литературы}

[1] A.K. Geim. Science, 324, 1530 (2009).

[2] К.С. Новоселов. УФН, 181 (12), 1304 (2011).

[3] B. Kuzmenko, E. van Heumen, F. Carbone, D. van der Marel. Phys Rev. Lett., 100, 117401 (2008).

[4] R.R. Nair, P. Blake, A.N. Grigorenko, K.S. Novoselov, T.J. Booth, T. Stauber, N.M.R. Peres, A.K. Geim. Science, 320, 1308 (2008).

[5] K.F. Mak, M.Y. Sfeir, Ya. Wu, C.H. Lui, J.A. Misewich, T.F. Heinz. Phys. Rev. Lett., 101, 196405 (2008).

[6] Е.В. Рутьков, Н.Р. Галль. Письма ЖЭТФ, 100 (10), 708 (2014).

[7] N.R. Gall, E.V. Rut'kov, A.Ya. Tontegode. Int. J. Mod. Phys. B, 11 (16), 1865 (1997).

[8] Е.В. Рутьков, А.В. Кузьмичев, Н.Р.Галль. Письма ЖЭТФ, 93 (3), 166 (2011).

[9] Z. Klusek, W. Kozlowski, S. Patta, I.S. Burnell-Gray, Z. Wagar, I.V. Makarenko, N.R. Gall, E.V. Rut'kov, A.Ya. Tontegode, A.N. Titkov. Appl. Surf. Sci., 252 (5), 1221 (2005).

[10] E.V. Rut'kov, N.R. Gall. In: Physics and Applications of Graphene - Experiments, ed. by S. Mikhailov (InTech, Rijeka, Croatia, 2011).

[11] Z.H. Ni et al. ACS Nano, 2, 2301 (2008).

[12] Т. Карлсон. Фотоэлектронная и оже-спектроскопия (Л., Машиностроение, 1981).

[13] E.V. Rut'kov, N.R. Gall. Appl. Surf. Sci., 300, 1087 (2014).

[14] E.V. Rut'kov, N.R. Gall. Surf. Sci., 645, 63 (2016).

[15] А.Н. Гордов. Основы пирометрирования (М., Металлургия, 1971).

[16] А.Я. Тонтегоде, Ф.К. Юсуфов. ЖТФ, 43, 1106 (1973).

[17] Z.Q. Li, E.A. Henriksen, Z. Jiang, Z. Hao, M.C. Martin, P. Kim, H.L. Stormer, D.N. Basov. Nature Phys., 4, 532 (2008).

[18] A. Pachoud, M. Jaiswal, P.K. Ang, K.P. Loh, B. Özyilmaz. Europhys. Lett., 92, 27001 (2010).

[19] В. Эсле. Технология электровакуумных материалов (М.-Л., Гос. энерг. изд-во, 1962) т. 1.

Редактор Л.В. Шаронова 
Optical transparency of ghaphene layers grown on a metal surface

E.V.Rut'kov ${ }^{1,2}$, N.P. Lavrovskaya ${ }^{2}$, E.S. Sheshenya ${ }^{1}$, N.R. Gall ${ }^{1,3}$

${ }^{1}$ Ioffe Institute,

194021 St. Petersburg, Russia

${ }^{2}$ Saint-Petersburg State University

of Aerospace Instrumentation,

190000 St. Petersburg, Russia

${ }^{3}$ Institute for Analytical Instrumentation,

Russian Academy of Sciences,

198095 St. Petersburg, Russia

Abstract It has been shown that the graphene film grown on a metal surface is transparent to visible range electromagnetic radiation emitted by the sample, up to the thickness of $\sim(12-15)$ layers. Such a film does not affect the brightness and actual sample temperatures, in contradiction with fundamental results on optical absorption of free graphene. At a higher thickness, the absorption agrees with the generally accepted concepts for free graphene. The effect has been observed also on $\operatorname{Rh}(111), \operatorname{Pt}(111), \operatorname{Re}(10 \overline{1} 0)$, and $\mathrm{Ni}(111)$, so one can consider it as being universal. We believe that the effect is due to change in graphene electronic features resulting from electron flow from metal. 